\title{
Foraging strategies of coexisting lacertid lizards in the arid Tankwa Karoo Basin of South Africa
}

\author{
I.J. du Plessis \& P. le F.N. Mouton* \\ Department of Botany and Zoology, University of Stellenbosch, Private Bag X1, \\ Matieland, 7602 South Africa \\ Received 13 October 2011. Accepted 8 May 2012
}

\begin{abstract}
Four lacertid lizards, Pedioplanis laticeps, P. lineo-ocellata, Meroles knoxii and Nucras tessellata, occur sympatrically on the arid plains of the Tankwa Karoo Basin in South Africa. The aim of this study was to evaluate the significance of foraging strategy in resource partitioning among the four species, allowing them to co-occur in a structurally simple system with a limited number of potential niches. Previous workers already identified P. lineo-ocellata and M. knoxii as sit-and-wait foragers and $N$. tessellata as an active forager. We recorded data on three foraging variables: movements per minute, proportion of time spent moving, and proportion of attacks on prey whilst moving, for juveniles and adults of $P$. laticeps. By comparing the foraging data obtained for $P$. laticeps to those for other lacertid species, we were able to demonstrate that adult $P$. laticeps are ambush foragers. We also noted a significant ontogenetic shift in foraging behaviour in P. laticeps, and, due to a significantly higher frequency of short brief movements, we classified juveniles as mixed foragers. The sharing of an ambush foraging strategy by at least three of the four lacertid species co-occurring on the Tankwa plains, suggests considerable overlap along the trophic dimension of ecological space. This overlap presumably promotes occupation of separate microhabitats by the three ambush foragers in the Tankwa Karoo Basin.
\end{abstract}

Key words: resource partitioning, foraging strategy, movements per minute, proportion of time spent moving, proportion of attacks on prey whilst moving, Pedioplanis laticeps

\section{INTRODUCTION}

For effective partitioning of resources and longterm coexistence, species should be well separated in ecological space defined by three main dimensions: trophic, spatial and temporal (Toft 1985). Foraging strategy is a component of the trophic dimension; species using different foraging strategies should be able to successfully partition or share the resources available to them within their communities (Pianka 1969; Pianka 1973; Hutchinson 1978; Perry et al. 1990). Successful resource partitioning decreases interspecific competition, increases feeding efficiency and increases the carrying capacity of an ecological system (Simon \& Middendorf 1976; Toft 1985; Perry et al. 1990). After the spatial dimension, Toft (1985) ranked the trophic dimension as the next most important ecological dimension within which the partitioning of resources takes place among lizards.

For lizards, two main foraging strategies are ambush foraging and active foraging (Pianka 1966). Lizards that use the ambush strategy hunt by lying in wait for prey, usually mobile and often fleet prey, e.g. grasshoppers and beetles, to move within striking range (Pianka 1971; McBrayer

*Author for correspondence. E-mail: pnm@sun.ac.za
2004; Cooper 2005). Ambush foragers rely on a sudden short burst of speed to catch prey items (Huey \& Pianka 1981). In contrast, active foragers rely more on endurance as they move through the habitat, actively searching for sedentary or patchy prey items, e.g. termites (Pianka 1971; Huey \&Pianka 1981; McBrayer 2004; Cooper 2005). Active foragers depend on visual surveillance and/or frequent tongue flicking to locate chemical cues, whereas ambush foragers typically rely on visual surveillance to locate potential prey items (Huey \& Pianka 1981; Cooper 1995, 1997, 2005, 2007; Cooper \& Whiting 1999). The realm of foraging strategy should, however, not be regarded as a dichotomous system, but rather a continuum within which ambush and active foragers represent the two extremes of the continuum (Pianka 1973; Magnusson et al. 1985; Pietruzka 1986; Perry et al. 1990; Cooper 2005; Miles et al. 2007). This view is overly simplified because foraging modes may be represented by spaces of several continuous dimensions (Cooper 2005).

Most species within entire lizard genera and even families exhibit the same foraging strategy (Huey \& Pianka 1981; Cooper \& Whiting 1999; Perry 1999). The Lacertidae is one of few excep- 
tions in this regard as there is evidence for at least two independent origins of ambush foraging in the family, for one of mixed foraging, and for a possible return from ambush foraging to active foraging (Cooper \& Whiting 1999).

The lizard communities of the Kalahari and Namibian deserts have been the focus of a number of ecological studies and, as a result, the foraging behaviour of several southern African lacertid lizard species has been recorded (Huey \& Pianka 1981; Cooper \& Whiting 1999). No information is, however, available on resource partitioning among lacertid lizards inhabiting the Tankwa Karoo Basin, one of the most arid regions in South Africa. Mean annual precipitation varies between 40 and 110 mm (Rubin 1998; Mucina \& Rutherford 2006) and temperatures between an average minimum of $5.7^{\circ} \mathrm{C}$ during winter and an average maximum of $35.9^{\circ} \mathrm{C}$ during summer (Rubin 1998).

Meyer et al. (2010) identified no less than seven terrestrial, diurnal lizard species occupying the Tankwa flats, four of which are lacertids. The four lacertids, Pedioplanis laticeps, P. lineo-ocellata, Meroles knoxii and Nucras tessellata, are very similar in body size and general morphology and are all classified as diurnal insectivores (Branch 1998). In studies conducted in the Kalahari and Namib desert systems, P. lineo-ocellata and M. knoxii were found to be sit-and-wait foragers and $N$. tessellata an active forager (Huey \& Pianka 1981; Cooper \& Whiting 1999). The aims of this study were firstly to determine the foraging strategy of P. laticeps, for which no information is available, and secondly to evaluate the contribution that differences in foraging strategy make to resource partitioning among the sympatric lacertid species in the Tankwa Karoo Basin.

\section{MATERIALS \& METHODS}

Information on foraging behaviour of Pedioplanis laticeps was obtained on the farm Gansfontein $\left(32^{\circ} 43^{\prime} 27.32^{\prime \prime} \mathrm{S}, 19^{\circ} 42^{\prime} 55.20^{\prime \prime} \mathrm{E}\right)$ in the southwestern parts of the Tankwa Karoo Basin, South Africa. Data were obtained during March and October 2008, on sunny days and at times and temperatures when lizards are known to be most active. The sampling procedure of Cooper \& Whiting (1999) was adopted in this study. Active lizards were located and observed by walking through the different habitat types where P. laticeps is known to occur (Du Plessis \& Mouton 2011). After the initial location of a lizard, the observer stopped to reduce any further disturbance of the lizard. If the lizard showed signs of nervousness due to the presence of the observer, the observer waited until the lizard appeared to behave normally again before any data were recorded. The standard procedure was to record all movement and foraging behaviour on a digital voice recorder. Only recordings that lasted longer than a minute and a half were included in the data set. The maximum time per recording was 10 minutes. Behavioural aspects recorded included all movements and stops (stationary for longer than two seconds) in addition to all feeding attempts. For each feeding attempt it was noted whether the attempt was initiated from a stationary position or during movement. Postural changes in position were not recorded as foraging movements. After a successful recording, the observer moved to a different location to lower the chances of replication. Individuals were classified as adult or juvenile on the basis of size and colouration, brightly coloured individuals smaller than $\sim 45 \mathrm{~mm}$ in SVL were considered to be juveniles (Nkosi et al. 2004).

Two traditional foraging variables were calculated, namely 'proportion of time spent moving' (PTM) and 'movements per minute' (MPM) (Huey \& Pianka 1981). A third variable, 'proportion of attacks on prey whilst moving' (PAM), was included to reduce the chances of misinterpreting movement data for foraging behaviour and to increase accuracy in defining the foraging strategy used (Cooper \& Whiting 1999). MPM and PTM values for juveniles and adults were compared using the Mann-Whitney test for non-parametric data.

To allow comparison of recorded foraging data for southern African lacertid lizards, a scatter plot was prepared, with MPM and PTM as axes, for juvenile and adult $P$. laticeps, three additional Pedioplanis species, four Meroles species, Nucras tessellata and Heliobolus lugubris (Huey \& Pianka 1981; Cooper \& Whiting 1999). In the two-dimensional foraging space represented by MPM and PTM, true ambush foragers will occupy the foraging space close to the origin where both MPM and PTM are close to zero. Active foragers on the other hand will vary greatly in their MPM values, but will have much higher PTM values. The more similar the foraging strategy used by two species the closer the two species will lie within foraging space.

\section{RESULTS}

Observations were made for six juvenile and 18 adult individuals of $P$. laticeps. Average observa- 
Table 1. Foraging data for Pedioplanis laticeps. The table provides the mean values calculated for MPM (movements per minute), PTM (proportion of time spent moving) and PAM (proportion of attacks on prey whilst moving) for each of the age groups. The foraging data for P. lineo-ocellata, Meroles knoxii and Nucras tessellata are also included.

\begin{tabular}{|c|c|c|c|c|c|c|}
\hline \multirow[b]{2}{*}{ Species } & \multicolumn{2}{|c|}{ MPM } & \multicolumn{2}{|c|}{ PTM (\%) } & \multirow[t]{2}{*}{ PAM (\%) } & \multirow[b]{2}{*}{$n$} \\
\hline & Average & S.E. & Average & $\overline{\text { S.E. }}$ & & \\
\hline P. laticeps (adults) & 0.61 & 0.95 & 4.41 & 7.81 & 0 & 18 \\
\hline P. laticeps (juveniles) & 2.54 & 1.5 & 21.87 & 13.36 & 100 & 6 \\
\hline P. lineo-ocellata ${ }^{1}$ & 0.49 & - & 5.00 & - & 0 & 4 \\
\hline P. lineo-ocellata ${ }^{2}$ & 1.54 & - & 14.30 & - & - & 15 \\
\hline M. knoxii & 0.61 & - & 7.00 & - & 0 & 27 \\
\hline N. tessellata ${ }^{1}$ & 0.7 & - & 90.00 & - & 100 & 1 \\
\hline N. tessellata ${ }^{2}$ & 2.9 & - & 50.20 & - & - & 11 \\
\hline
\end{tabular}

${ }^{1}$ Cooper \& Whiting (1999).

${ }^{2}$ Huey \& Pianka (1981).

tion time for juveniles was 5.8 minutes and 8.8 minutes for adults (193 minutes total observation time). For adults, the mean MPM and PTM values recorded during the March $(n=6)$ and October $(n=12)$ sampling periods did not differ significantly and the two data sets were therefore combined (Mann-Whitney test: MPM: $z=-1.03$, $P=0.303$; PTM: $z=-0.89, P=0.374)$. The MPM and PTM values for juveniles were significantly higher than those for adults (Mann-Whitney test: MPM: $z=2.7, P=0.0069$; PTM: $z=2.83$, $P=0.0047 ;$ Table 1$)$. In total, five feeding attempts were observed. The two attempts by adult individuals were initiated from a stationary position, from the cover of a bush (PAM: 0\%), whereas the three attempts by juveniles were all made during movement (PAM: 100\%). Juvenile lizards were often seen actively searching for prey items, i.e. digging under rocks. In the two-dimensional foraging space defined by MPM and PTM, adult P. laticeps occupied a position close to those of $P$. lineoocellata, M. knoxii, and $M$. reticulatus, whereas juveniles occupied a position close to that of M. ctenodactylus (Fig. 1).

\section{DISCUSSION}

The relatively low MPM and PTM values that we recorded for adult $P$. laticeps would place this species at the ambush end of the ambush to active foraging continuum (Perry et al. 1990; Perry 1995; Cooper 2005). In fact, our data would place $P$. laticeps in close proximity to P. lineo-ocellata and Meroles knoxii (Fig. 1), two lacertids that have been classified as distinct ambush foragers in previous studies (Huey \& Pianka 1981; Cooper \& Whiting 1999). Prior to this study, foraging data were available for only three species in the genus Pedioplanis.
Pedioplanis namaquensis and P. undata were classified as active foragers (Cooper \& Whiting 1999) and $P$. lineo-ocellata as an ambush forager (Huey \& Pianka 1981; Cooper \& Whiting 1999). The classification of the latter species as an ambush forager is supported by its cranial morphology (McBrayer 2004), its relatively low stamina (Nagy et al. 1984), and its diet, which includes diurnally active prey items such as flies and beetles (Nagy et al. 1984). The results of our study show that ambush foraging may be more widespread in the genus.

In Pedioplanis, P. laticeps and P. burchelli form the basal clade, being the sister group to the rest of the genus (Makokha et al. 2007). Foraging data are presently not available for $P$. burchelli, but, with $P$. laticeps being an ambush forager, ambush foraging may well be the basal condition in Pedioplanis, as in Meroles (Huey \& Pianka 1981; Arnold 1991; Cooper \& Whiting 1999). The other identified ambush forager, $P$. lineo-ocellata, together with P. breviceps, occupies a basal position in the main Pedioplanis clade (Makokha et al. 2007), lending further support to the notion that ambush foraging may be the basal condition in the genus.

The limited PAM data that we recorded for adult P. laticeps seem to support the classification of this species as an ambush forager. A PAM value of $0.00 \%$ has also been recorded for P. lineo-ocellata, the other known ambush forager in the genus for which foraging data are available (Cooper \& Whiting 1999). Theoretically, feeding attempts by active foragers should occur at a higher frequency and should therefore be observed more often than for ambush foragers. Cooper \& Whiting (1999) gathered foraging data for 14 Heliobolus lugubris individuals during which 45 foraging attempts were observed, all of which were initiated whilst 


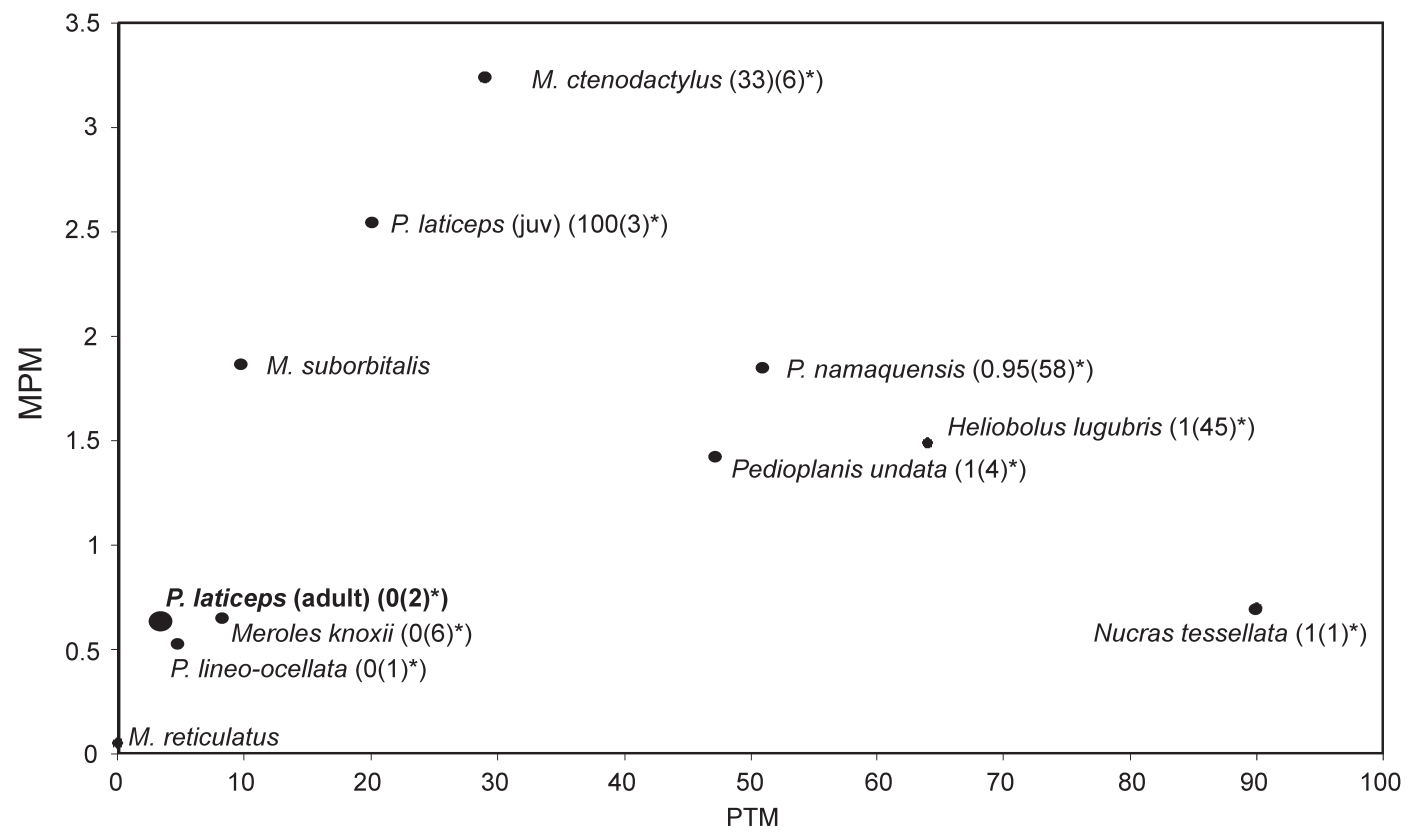

Fig. 1. Positions of juvenile and adult Pedioplanis laticeps relative to various other southern African lacertids (Huey \& Pianka 1981; Cooper \& Whiting 1999) in two-dimensional foraging space, defined by MPM and PTM. PAM (proportion of attacks on prey whilst moving) and the number of attacks witnessed $\left({ }^{*}\right)$ are provided for some species.

the specific lizard was on the move $(\mathrm{PAM}=100 \%)$. In total, $18 P$. laticeps adults were observed for 158 minutes, during which time only two feeding attempts were observed. This low number of feeding attempts can therefore be seen as an indication that this species uses an ambush foraging strategy, but it may also be and indication of food scarcity during the time of our survey.

The considerably higher MPM and PTM values recorded for juveniles than for adults of $P$. laticeps show that there is an ontogenetic shift in foraging strategy in this species. The values recorded for juvenile $P$. laticeps, would place them in close proximity to $M$. ctenodactylus in foraging space defined by MPM and PTM (Fig. 1). Cooper \& Whiting (1999) described M. ctenodactylus as a mixed forager, i.e. a species displaying a high frequency of brief movements during foraging, in contrast to prolonged movement typical of active foragers or no or only a few movements typical of ambush foragers.

A difference in foraging strategy between adults and juveniles has been recorded in several other lizard species. In some Australian species, juvenile individuals tend to target smaller prey items than adult individuals (Pianka 1969). Robson \& Lambert (1980) found an ontogenetic shift in food preferences in Acanthodactylus boskianus of southern
Morocco and stated that this could be accompanied by a similar shift in foraging strategy. Likewise Hawlena et al. (2006) found that juveniles of A. beershebensis display a more active foraging strategy than adults.

Correlating daily intake of chemical potential energy with growth rate, Nagy et al. (1984) found that the known active forager (H. lugubris) grew twice as fast during the same time as the ambush forager (P. lineo-ocellata). Increased energy demand might force the juveniles to adopt a more active foraging strategy, enabling them to sustain a certain required growth rate. Hawlena et al. (2006) suggested that the more active foraging behaviour of $A$. beershebensis juveniles could be a mechanism to sustain an increased growth rate, which results in an increase in absolute adult fitness. Juvenile foraging behaviour such as increased movements and active searching in the form of digging could be seen as a behavioural response to adapt to a limiting foraging environment.

Similar to the situation in many other lacertid species where juveniles differ from adults in colouration (Branch 1998), the colouration of juvenile $P$. laticeps is markedly different from that of adults. Juveniles are dark in colour, with yellowish dorsal stripes over the length of the trunk and a red-orange tail. Adults on the other hand, have a 
much more faded appearance with spots and faint stripes. In many lacertid and teiid species, juveniles have bright tail colours combined with light longitudinal stripes on a dark skin surface that fade into more cryptic colour patterns as the individual matures (Cooper \& Greenberg 1992). It has been suggested that ontogenetic colour change is a response to a change in the predator suite that preys on the different age groups of a species (Cooper \& Vitt 1985). More active species tend to be striped, speckled or uniformly coloured, while ambush foragers are known to be blotchier in colouration for better concealment (Cooper \& Greenberg 1992).

The finding that $P$. laticeps, P. lineo-ocellata and $M$. knoxii, three of the four lacertid species cooccurring in the arid Tankwa Karoo Basin of South Africa, are all typical ambush foragers, suggests considerable overlap along the trophic dimension of ecological space. The spatial separation of the three species due to different microhabitat preferences (Du Plessis \& Mouton 2011), however, allows the narrow sympatric occurrence of the three species within the Basin. Similar results were obtained in previous studies in the Kalahari desert where as many as three out of four active foragers (Heliobolus lugubris, Pedioplanis namaquensis, Nucras tessellata and N. intertexta) are able to occur sympatrically due to different microhabitat preferences (Pianka 1971). Within other desert systems with limited microhabitat heterogeneity, a significant overlap along the trophic dimension led to the temporal separation of two sympatric species (Rouag et al. 2007). The fact that $P$. laticeps, P. lineoocellata and M. knoxii are spatially separated at the study site, allows them to have a similar foraging strategy.

\section{ACKNOWLEDGEMENTS}

We thank D. Kershoff, owner of the farm Gansfontein, for allowing us to use his farm as study site; Jessica Cloete, Jeannie Hayward, Anita Meyer, Simon Capon, Mat Lacheneicht, AnneMarie du Plessis, Anneke du Plessis, Kate Price and Jacobie and Yolandé Mouton for assistance in the field; and the National Research Foundation (NRF) and Critical Ecosystems Partnership Fund (CEPF) for funding.

\section{REFERENCES}

ARNOLD, E.N. 1991. Relationships of the South African lizards assigned to Aporosaura, Meroles and Pedioplanis (Reptilia: Lacertidae). Journal of Natural History 25: 783-807.
BRANCH, W.R. 1998. Field Guide to Snakes and Other Reptiles of Southern Africa. Struik Publishers, Cape Town.

COOPER, W.E., JR. 1995. Foraging mode, prey chemical discrimination, and phylogeny in lizards. Animal Behavior 50: 973-985.

COOPER, W.E., Jr. 1997. Correlated evolution of prey chemical discrimination with foraging, lingual morphology, and vomeronasal chemoreceptor abundance in lizards. Behavioral Ecology and Sociobiology 41: 257-265.

COOPER, W.E., Jr. 2005. Foraging mode controversy: both continuous variation and clustering of foraging movements occur. Journal of Zoology, London 267: 179-190.

COOPER, W.E., Jr. 2007. Lizard chemical senses, chemosensory behavior, and foraging mode. In: Lizard Ecology: The Evolutionary Consequences of Foraging Mode, (eds) S.M. Reilly, L.D. McBrayer \& D.B. Miles, pp. 237-270. Cambridge University Press, Cambridge.

COOPER, W.E., Jr. \& GREENBERG, N. 1992. Reptilian colouration and behaviour. In: Hormones, Brain and Behaviour, Biology of Reptilia, (eds) C. Gans \& D. Crews, Vol. 18, pp. 298-422. University of Chicago Press, Chicago.

COOPER, W.E., Jr. \& VITT, L.J. 1985. Blue tails and autotomy: enhancement of predation avoidance in juvenile skinks. Zeitschrift für Tierpsychologie 70: 265-276.

COOPER, W.E., Jr. \& WHITING, M.J. 1999. Foraging strategies of South African lacertid lizard species. Amphibia-Reptilia 20: 299-311.

DU PLESSIS, I.J. \& MOUTON, P. LE F.N. 2011. Habitat preferences of three sympatric lacertid lizards in the arid Tankwa Karoo Basin of South Africa. African Zoology 46: 88-94.

HAWLENA, D., BOOCHNIK, R., ABRAMSKY, Z. \& BOUSKILA, A. 2006. Blue tail and striped body: why do lizards change their infant costume when growing up? Behavioural Ecology 17:889-896.

HUEY, R.B. \& PIANKA, E.R. 1981. Ecological consequences of foraging mode. Ecology 62: 991-999.

HUTCHINSON, G.E. 1978. An Introduction to Population Ecology. Yale University Press, New Haven.

MAGNUSSON, W.E., JUNQUEIRA DE PAIVA, L., MOREIRA DE ROCHA, R., FRANKE, C.R., KASPER, L.A. \& LIMA, A.P. 1985. The correlates of foraging mode in a community of Brazilian lizards. Herpetologica 41: 324-332.

MAKOKHA, S.J., BAUER, A.M., MAYER, W. \& MATTHEE, C.A. 2007. Nuclear and mtDNA-based phylogeny of southern African lizards, Pedioplanis (Sauria: Lacertidae). Molecular Phylogenetics and Evolution 44: 622-633.

McBRYAER, L.D. 2004. The relationship between skull morphology, biting performance and foraging mode in Kalahari lacertid lizards. Zoological Journal of the Linnean Society 140: 403-416.

MEYER, A., MOUTON, P. LE F.N. \& MUCINA, L. 2010. The biogeographical influence of the Tankwa Karoo Basin on reptile distribution in south-western South Africa. African Journal of Herpetology 59: 53-64.

MILES, D.B, LOSOS, J.B. \& IRSCHICK, D.J. 2007. Morphology, performance and foraging mode. In: Lizard Ecology: The Evolutionary Consequences of Foraging Mode. (eds) S.M. Reilly, L.D. McBrayer \& D.B. 
Miles, pp. 49-93. Cambridge University Press. Cambridge.

MUCINA, L. \& RUTHERFORD, M.C. (eds) 2006. The vegetation of South Africa, Lesotho and Swaziland. Strelitzia 19. South African National Biodiversity Institute, Pretoria.

NAGY, K.A., HUEY, R.B. \& BENNETT, A.F. 1984. Field energetics and foraging mode of Kalahari lacertid lizards. Ecology 65: 588-596.

NKOSI, W.T., HEIDEMAN, J.L. \& VAN WYK, J.H. 2004. Reproduction and sexual dimorphism in the lacertid lizard Pedioplanis burchelli (Sauria: Lacertidae) in South Africa. Journal of Herpetology 38: 473-480.

PERRY, G. 1995. The evolutionary ecology of lizard foraging: a comparative study. Ph.D. thesis, University of Texas, Austin.

PERRY, G. 1999. The evolution of search modes: ecological versus phylogenetic perspectives. American Naturalist 153: 99-109.

PERRY, G., LAMPL, I., LERNER, A., ROTHENSTEIN, D., SHANI, E., SIVAN, N. \& WERNER, Y.L. 1990. Foraging mode in lacertid lizards: variation and correlates. Amphibia-Reptilia 11: 373-384.

PIANKA, E.R. 1966. Convexity, desert lizards and spatial heterogeneity. Ecology 47: 1055-1059.
PIANKA, E.R. 1969. Sympatry of desert lizards (Ctenotus) in Western Australia. Ecology 50: 1012-1030.

PIANKA, E.R. 1971. Lizard species density in Kalahari Desert. Ecology 52: 1024-1029.

PIANKA, E.R. 1973. Structure of lizard communities. Ecology and Systematics 4: 53-57.

PIETRUZKA, R.D. 1986. Search tactics of desert lizards: how polarized are they. Animal Behaviour 34: 17421758.

ROBSON, G.M. \& LAMBERT, M.R.K. 1980. Observations on the insect food of some semi-desert lizards in southern Morocco. Journal of Arid Environments 3: 141-151.

ROUAG, R., DJILALI, H., GUERAICHI, H. \& LUISELLI, L. 2007. Resource partitioning patterns between two sympatric lizard species from Algeria. Journal of Arid Environments 69: 158-168.

RUBIN, F. 1998.The physical environment and major plant communities of the Tankwa National Park. Koedoe 41: 61-94.

SIMON, C.A. \& MIDDENDORF, G.A. 1976. Resource partitioning by an iguanid lizard: temporal and microhabitat aspects. Ecology 57: 1317-1320.

TOFT, C.A. 1985. Resource partitioning in amphibians and reptiles. Copeia 1985: 1-21.

Responsible Editor: J.H. van Wyk 